\title{
Functional complementation of
} pop2-3 T-DNA insertion mutant of Arabidopsis thaliana by overexpression of gamma-aminobutyric acid transaminase gene to reveals its role in complex pattern of leaf senescence

\author{
Syed Uzma Jalil', Mohammad Israil Ansari ${ }^{1,2}$ \\ ${ }^{1}$ Amity Institute of Biotechnology, Amity University Uttar Pradesh, Lucknow Campus, Lucknow, Uttar Pradesh, \\ India, ${ }^{2}$ Department of Botany, University of Lucknow, Lucknow, Uttar Pradesh, India
}

\section{Abstract}

Aim: Gamma-aminobutyric acid (GABA) is the chief inhibitory neurotransmitter in the mammalian central nervous system, and its important role is reducing the neuronal excitability throughout the nervous system. The GABA transaminase (GABA-T) enzyme catalyzes the transamination of GABA to form succinic semialdehyde using pyruvate as amino acid acceptor in GABA shunt pathway. The Arabidopsis thaliana GABA-T gene (pollenpistil incompatibility 2 [POP2]; At3g22200) belongs to Class III pyridoxal-phosphate-dependent aminotransferase family. The full-length cDNA of the gene encodes a protein of 513 amino acids residues and located at chromosome 3. It could be demonstrated that the GABA-T is involved in the regulation of leaf senescence in $A$. thaliana. Materials and Methods: To ensure the role of GABA-T gene in leaf senescence, we have performed functional complementation using transformation of pPZP200GB-GABA-T construct into pop 2-3 T-DNA insertion mutant (GABA-T knockout mutant) of A. thaliana (GABA-T complement plant) that the mutant plant reverts back to wild-type plant. We have measured and compared the physiological characteristics associated with senescence (chlorophyll content, ion leakage and lipid peroxidation) and quantified the GABA shunt components (GABA content, GABA-T, and glutamate decarboxylase activity) of the wild-type, pop 2-3 mutant, and GABA-T complement plant of $A$. thaliana. Results: The result showed the low chlorophyll content, increased ion leakage, and higher level of lipid peroxidation in pop2-3 mutant than wild-type plant after 30 days in normal growth condition. GABA-T pop 2-3 mutants act as signals that mediate early leaf senescence. GABA-T complement plant shared similar characteristics of senescence parameters with wild-type plants. Conclusion: These data concluded that GABA-T is involved in the regulation of leaf senescence in $A$. thaliana.

Key words: Complementation, gamma-aminobutyric acid-transaminase, senescence, T-DNA insertion mutant

\section{INTRODUCTION}

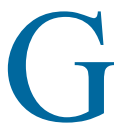
amma-aminobutyric acid (GABA) is a ubiquitous non-protein amino acid and found in prokaryotes and eukaryotes. ${ }^{[1]}$ GABA has received increased attention in research due to its role as an inhibitory neurotransmitter in the mammalian central nervous system by binding to specific transmembrane receptors in the plasma membrane of both pre- and post-synaptic neuronal processes. ${ }^{[2,3]}$ In humans, GABA is also directly responsible for the regulation of muscle tone, and it regulates many of the depressive and sedative actions in brain tissue and is critical for relaxation. ${ }^{[2-4]}$ Rapid GABA accumulation has been reported in plants under biotic and abiotic stress conditions ${ }^{[5]}$ and the

Address for Correspondence:

Dr. Mohammad Israil Ansari, Department of Botany, University of Lucknow, Lucknow - 226007 , Uttar Pradesh, India. Phone: +91-9839541698.

E-mail: ansari_mi@lkouniv.ac.in

Received: 11-08-2017

Revised: $25-08-2017$

Accepted: 28-08-2017 
evolvement of GABA-shunt in responses to abiotic stress has been reported by Bouché and Fromm ${ }^{[1]}$ and Ludewig et al. ${ }^{[6]}$ These stresses initiate a signal transduction pathway, which increased cytosolic calmodulin-dependent activity of the glutamate decarboxylase (GAD). However, it appears that GABA is important for vegetative development. GABA is catabolized through GABA transaminase (GABA-T) to succinic semialdehyde (SSA), which in turn is oxidized through a NAD-dependent SSA dehydrogenase (SSADH) to succinate.

GABA-T (EC 2.6.1.19) is a key enzyme in GABA shunt that involved in the carbon and nitrogen metabolism in plants. The GABA-T encoding gene (At3g22200) is present as a single copy in the Arabidopsis genome and was initially termed pollen-pistil incompatibility 2 (POP2). ${ }^{[7]}$ The GABA-T enzyme catalyzing the reversible transamination of GABA with either pyruvate or 2-oxoglutarate as an amino acceptor to form SSA and either alanine or glutamate ${ }^{[8]} A$. thaliana GABA-T knockout pop 2-3 has T-DNA insertion mutation at intron 14, and T-DNA insertion plants are indistinguishable from wild types during vegetative growth. ${ }^{[7,9]}$

During the process of senescence, some protein will be degraded into $\alpha$-amino acid with the assistance of proteases. The ammonia released from the transamination of $\alpha$-amino acid will be used to convert it into glutamate and $\alpha$-keto acids with the assistance of enzyme glutamine synthetase into glutamine. Further, this glutamine with enzyme asparagine synthetase will be converted into asparagine ${ }^{\left[10^{-12]}\right.}$ GABA is produced in cytosol through the decarboxylation of glutamate in pathway catalyzed by GAD. The process of GABA to glutamate transformation accelerates when there is restraint of protein synthesis. ${ }^{[12,13]}$ The metabolism of GABA is identified with glutamate metabolism in senescence process, and then, the glutamate will go into GABA shunt. This GABA is transaminated to SSA through GABA-T; thus, SSA is oxidized to succinate through SSADH in mitochondria and enter into tricarboxylic acid cycle. Therefore, GABA-shunt pathway converts glutamate into succinate through GABA in the plant leaf senescence and nitrogen assimilation. ${ }^{[1,14]}$

We have previously published the physiological and metabolic characterization of the GABA-T pop 2-3 mutant on different stress responses. Based on screening of senescence parameters, we showed that knockout of the GABA-T causes enhanced accumulation of reactive oxygen species and cell death in response to different stress conditions. ${ }^{[15]}$ In the present study, we have investigated the function of $A$. thaliana GABA-T gene in leaf senescence by functional complementation of GABA-T into pop2-3 T-DNA insertion mutant in conjunction with GABA-shunt component analysis. We demonstrate here that pop2-3 T-DNA insertion mutant expressed early leaf senescence than wild type, based on screening of senescence parameters. A comparative analysis in the pop 2-3 mutant, wild-type and GABA-T complement plant reveals that GABA-T controls both cellular protection activities and senescence activities and involved in the regulation of leaf senescence process.

\section{MATERIALS AND METHODS}

\section{Plant Materials and Growth Conditions}

A. thaliana Landsberg erecta ecotype (Ler) were used as wild type, and $A$. thaliana pop2-3 homozygous T-DNA insertion mutant plants for GABA-T (Stock name CS6387) obtained from Arabidopsis Biological Resource Center (Ohio State University, USA) were find out using polymerase chain reaction (PCR) with primer from the left and right border of T-DNA and primer from flanking region. Plants were grown at $22^{\circ} \mathrm{C}$ for long-day condition ( $16 \mathrm{~h} \mathrm{light} / 8 \mathrm{~h}$ dark cycle) on the soil. For soil growth, seeds were sown in Bio-Mix Potting Substratum (Keltech Energies Ltd. India) and placed at $4^{\circ} \mathrm{C}$ for 4 days in the dark to break residual dormancy and later transferred to normal growth conditions.

\section{RNA Isolation and Reverse Transcriptional PCR (RT-PCR)}

Total RNA for RT-PCR was isolated from the 3-week-old leaf of $A$. thaliana by RNA extraction mini kit (Promega, Madison, WI) according to the manufacturer's instructions. cDNA synthesis (using SuperScript III Reverse Transcriptase) was done as described by the supplier (Invitrogen, Karlsruhe, Germany). The cDNA was diluted 25 times and used to amplify the GABA-T gene using gene-specific primers as described by Miyashita and Good, Al-Quran and Share. ${ }^{[16,17]}$

\section{Generation of Plant Transformation Construct and Arabidopsis Transformation for Complementation}

Coding region of $A$. thaliana GABA-T gene (GenBank accession number AF351125) was cloned into binary vector pPZP200GB using XbaI and SacI restriction enzyme by replacing the $\beta$-glucuronidase fragment. This pPZP200GB with $\beta$-glucuronidase and BAR (BASTA resistance gene) cassettes was derived from pBI221 (Clontech Laboratories, Palo Alto, CA) and pSK-35S-BAR. ${ }^{[18]}$ The obtained plasmid construct was named pPZP200GB-GABA-T. The pPZP200GB-GABA-T construct was transformed into Agrobacterium tumefaciens strain C-58 by electroporation. A. thaliana GABA-T T-DNA insertion mutant (Stock name CS6387) was transformed through Agrobacterium-mediated transformation by floral-dipping method. ${ }^{[19]}$ Transgenic plants were selected by spraying seedlings at 7, 9, and 11 days after germination with a solution of $0.4 \%$ of BASTA herbicide. ${ }^{[20]} \mathrm{T} 2$ generations were selected for isolating homozygous lines. 


\section{Analysis of Leaf Senescence Parameters}

All parameters of leaf senescence (total chlorophyll content, electrolyte leakage, and lipid peroxidation) were measured with the fifth rosette leaves, which were harvested at specified days from different developmental stages (10-, 20-, and 30-day-old plant of $A$. thaliana).

\section{Measurement of Total Chlorophyll}

Total chlorophyll content was isolated from the leaves of pop 2-3 mutant, wild-type, and complement plants from $10-, 20-$, and 30-day-old plant and measured as per milligram fresh weight of tissue. ${ }^{[21]}$

\section{Measurement of Membrane Lon Leakage}

Leaves material of pop2-3 mutant, wild-type, and complement plants at different developmental stages (10-, 20-, and 30-day-old plant) were used for the determination of membrane ion leakage according to Chen et al. ${ }^{[22]}$

\section{Quantification of Malondialdehyde (MDA)}

The extent of lipid peroxidation was estimated by determining the concentration of MDA from leaf material (FW) of pop 2-3 mutant, wild-type, and complement plants at different developmental stages (10-, 20-, and 30-day-old plant) according to Heath and Packer. ${ }^{[23]}$

\section{Quantification of GABA Content}

GABA was extracted and determined from frozen tissues of different developmental stages (10-, 20-, and 30-day-old plant) of wild-type, pop 2-3 mutants, and complement plants; the GABA content in the sample was calculated based on the difference in absorbance. The standard GABA was prepared in micromole concentration and used for constructing the calibration graph as done in the previous study by Jalil et al. ${ }^{[15]}$

\section{Determination of Enzyme (GABA-T and GAD) Activities}

GABA-T and GAD activities assay were performed from the protein that was extracted from the leaves of wild type, pop 2-3 mutants, and complement plants of $A$. thaliana at different stages (10-, 20-, and 30-day-old plant) as described earlier by Jalil et al. ${ }^{[15]}$

\section{Statistical Analysis}

Statistical analysis was carried out using Microsoft excel, and each data point was expressed as mean \pm standard deviation of three independent experiments.

\section{RESULTS}

\section{Comparative Analysis of Physiological Parameters of Leaf Senescence}

To confirmed the physiological state of the complement plants, chlorophyll content, ion leakage, and lipid peroxidation of the pop 2-3 mutant, wild-type, and complement plants leaves at different developmental stages (10-, 20-, and 30-day-old plants) were measured. We first examined the chlorophyll content of wild-type, pop2-3 mutants, and complement plants, and we observed that the chlorophyll content of pop 2-3 mutants in 10, 20, and 30 days $(1.29 \pm 0.01,1.28 \pm 0.01$, and $0.63 \pm 0.09 / \mathrm{mg}$, respectively) were less than the wildtype plants $(1.97 \pm 0.01,1.92 \pm 0.02$, and $1.79 \pm 0.10 / \mathrm{mg}$, respectively). Chlorophyll content of pop2-3 mutant was stable up to 20 days but decreased from 30 days, whereas chlorophyll content in wild-type plant was almost stable up to 30 days. The chlorophyll content of complement plant was approximately similar to wild-type plant and stable up to 30 days $(1.86 \pm 0.1,1.79 \pm 0.1$, and $1.67 \pm 0.06 / \mathrm{mg}$, respectively) as shown in Figure 1a.

Ion leakage in pop 2-3 mutant was stable up to 20 days from the date of emergence ( 10 days old $16.97 \pm 0.01 \%$ and 20 days old $17.34 \pm 0.001 \%$ ) and began to sharply increase from 30 days $(54.37 \pm 0.05 \%)$. However, ion leakage in wild-type leaves stable up to 20 days $(16.01 \pm 0.004 \%$ and $16.94 \pm 0.006 \%$ ) and slightly increased from 30 -day-old plant $(19.36 \pm 0.024 \%)$ and the ion leakage of complement plant were approximately similar to wild-type plant, and there was no increase in the ratio throughout 30 days in complement plant $(16.27 \pm 0.03 \%, 17.09 \pm 0.05 \%$, and $17.93 \pm 0.03 \%$, respectively) as shown in Figure $1 \mathrm{~b}$.

The MDA content in the pop 2-3 mutant was slightly greater than wild-type plant and stable up to 20 days $(0.014 \pm 0.001$ and $0.015 \pm 0.003 \mu \mathrm{M} / \mathrm{mg}$, respectively) and start to increases from 30-day-old plants $(0.023 \pm 0.001 \mu \mathrm{M} / \mathrm{mg})$. Whereas, in wild-type plants, MDA content was stable from 10 to 30 days $(0.012 \pm 0.002,0.013 \pm 0.001$, and $0.014 \pm 0.001 \mu \mathrm{M} / \mathrm{mg}$, respectively). The MDA content of GABA-T complement plant was approximately similar to wild-type plant and stable up to 30 days $(0.012 \pm 0.003,0.013 \pm 0.001$, and $0.013 \pm 0.003 \mu \mathrm{M} / \mathrm{mg}$, respectively) as shown in Figure 1c.

\section{Quantification of GABA Content from Leaves of Wild-type, pop2-3 Mutant, and GABA-T Complement Plants}

A targeted analysis of GABA content in GABA-T complement, pop2-3 mutant, and wild-type plants was performed and showed that GABA content of pop2-3 mutant leaves was much higher than wild-type plant. GABA content in pop 2-3 mutant was stable up to 20-day-old plants (10 days $0.10 \pm 0.03$ and 20 days $0.11 \pm 0.05 \mu \mathrm{moles} / \mathrm{g} \mathrm{FW}$ ) 


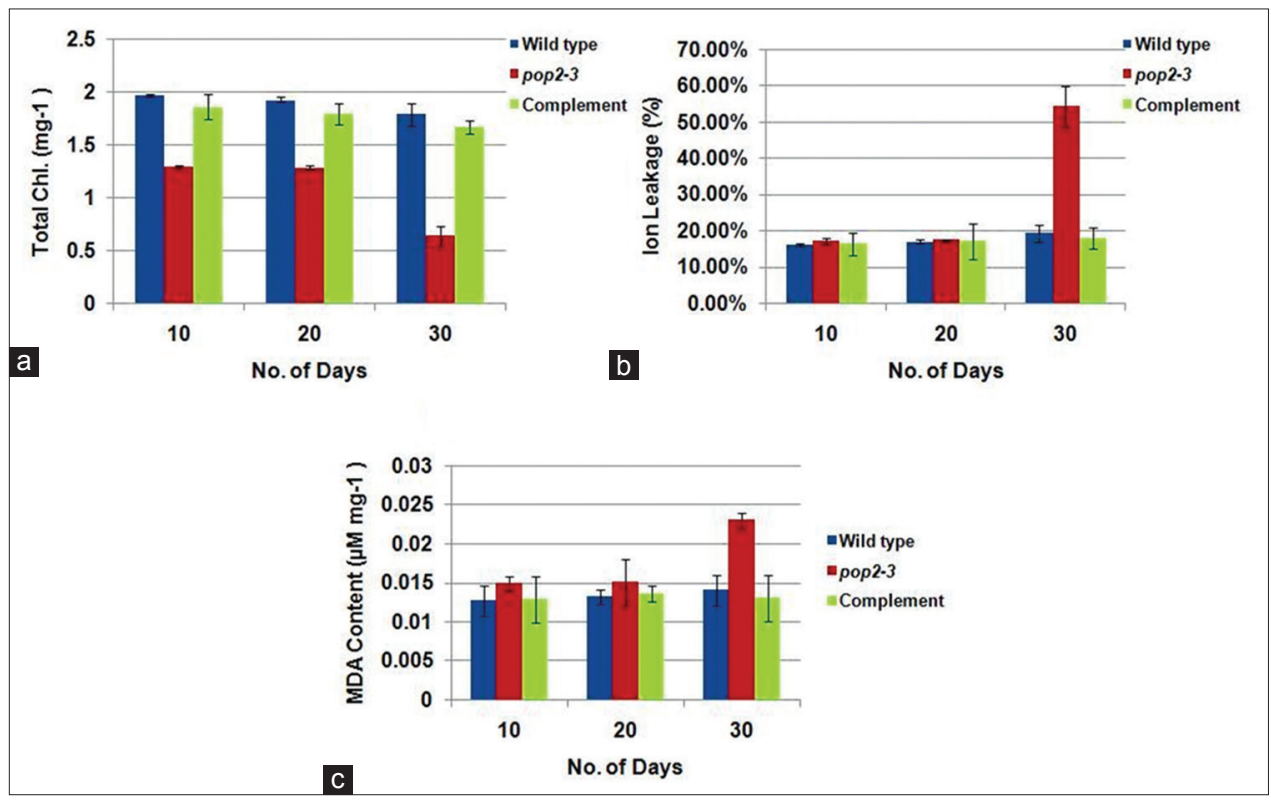

Figure 1: Physiological parameter of leaf senescence of pop2-3 mutant, gamma-aminobutyric acid transaminase (GABA-T) complement, and wild-type plant of Arabidopsis thaliana. (a) Determination of chlorophyll content of wild-type, GABA-T complement, and pop2-3 mutant leaves, (b) determination of ion leakage of wild-type, mutant, and GABA-T complement leaves, (c) Quantification of lipid peroxidation by the level of malondialdehyde wild-type, GABA-T complement, and mutant leaves. Data represent mean \pm standard deviation of 3-5 biological replicates

and sharply increases from 30 days $(0.46 \pm 0.07 \mu$ moles $/ g$ FW), whereas in wild type, it was approximately stable up to 30 days $(0.18 \pm 0.03,0.19 \pm 0.04$, and $0.21 \pm 0.03 \mu$ moles $/ g$ FW, respectively). GABA content of GABA-T complement plant was approximately similar to wild-type plant and stable up to 30 days $(0.19 \pm 0.05,0.22 \pm 0.03$, and $0.24 \pm 0.01$ $\mu$ moles/g FW, respectively) as shown in Figure 2.

\section{DETERMINATION OF ENZYME ACTIVITY (GABA-T AND GAD)}

Enzyme activity of GABA-T and GAD was assayed and compared during 10-, 20-, and 30-day-old plants of $A$. thaliana wild type, pop2-3 mutant, and GABA-T complement plants [Figure $3 \mathrm{a}$ and $\mathrm{b}$ ]. The enzyme activity (GABA-T and GAD) was much more in wild-type plants than in pop2-3 mutants. The enzymes (GABA-T and GAD) activity of pop 2-3 mutant were stable till 20 days (GABA-T in 10 days $2.34 \pm 0.54,20$ days $2.89 \pm 0.37 \mathrm{nmoles} / \mathrm{min} / \mathrm{mg}$ protein, and GAD in 10 days $10.09 \pm 0.6,20$ days $10.27 \pm 0.51 \mathrm{nmoles} / \mathrm{min} / \mathrm{mg}$ protein), whereas it was stable till 30 days in wild-type plant (GABA-T $12.28 \pm 0.36,12.92 \pm 0.57$, and $13.67 \pm 0.44 \mathrm{nmoles} / \mathrm{min} /$ mg protein, respectively, and GAD $20.97 \pm 0.8,21.08 \pm 0.7$, and $22.37 \pm 0.83 \mathrm{nmoles} / \mathrm{min} / \mathrm{mg}$ protein, respectively). The enzymes (GABA-T and GAD) activity of GABA-T complement plant was approximately similar to wild-type plant and stable up to 30 days (GABA-T $12.28 \pm 0.36$, $12.92 \pm 0.57$, and $13.76 \pm 0.68 \mathrm{nmoles} / \mathrm{min} / \mathrm{mg}$ protein, respectively, and GAD $18.86 \pm 0.72,19.32 \pm 1.68$, and $20.97 \pm 0.91 \mathrm{nmoles} / \mathrm{min} / \mathrm{mg}$ protein, respectively) as shown in Figure $3 a$ and $b$.

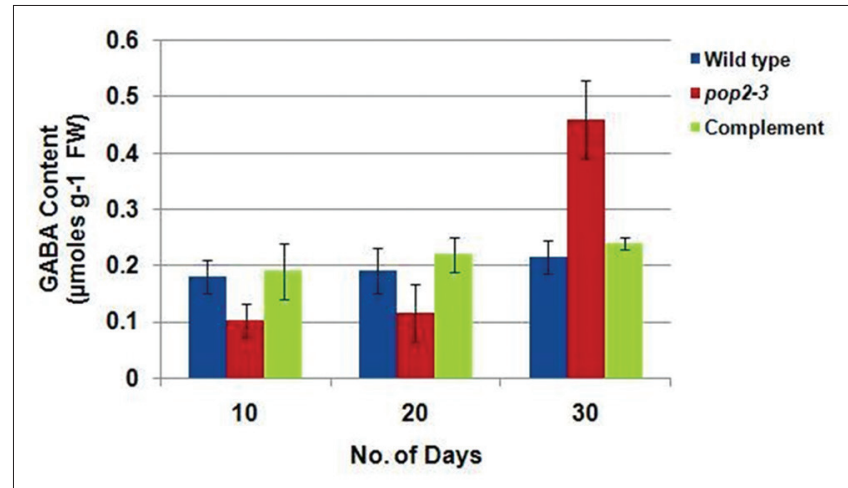

Figure 2: Determination of gamma-aminobutyric acid (GABA) content of pop2-3 mutant, GABA transaminase complement, and wild type leaves of Arabidopsis thaliana. Data represent mean \pm standard deviation of 3-5 biological replicates

\section{DISCUSSION}

Leaf senescence is dependent on age and developmental stage under normal conditions, and in the absence of external stress, the symptoms of senescence are normally observed in old leaves. Involvement of GABA-T gene in regulation of carbon/nitrogen metabolism and plant development has been reported by several investigators. ${ }^{[8,24,25]}$ It has been also studied that wild type of $A$. thaliana is more tolerant to oxidative stress, as compared to pop 2-3 mutant of GABA-T stress, and morphology of pop 2-3 mutant might differ to wild type plants..$^{[9,15,17,26]}$ The oversensitivity of the pop2-3 mutants to the abiotic and oxidative stress treatment indicates that a GABA-T defect impaired GABA accumulation that might be required for growth and implicates that GABA is involved 


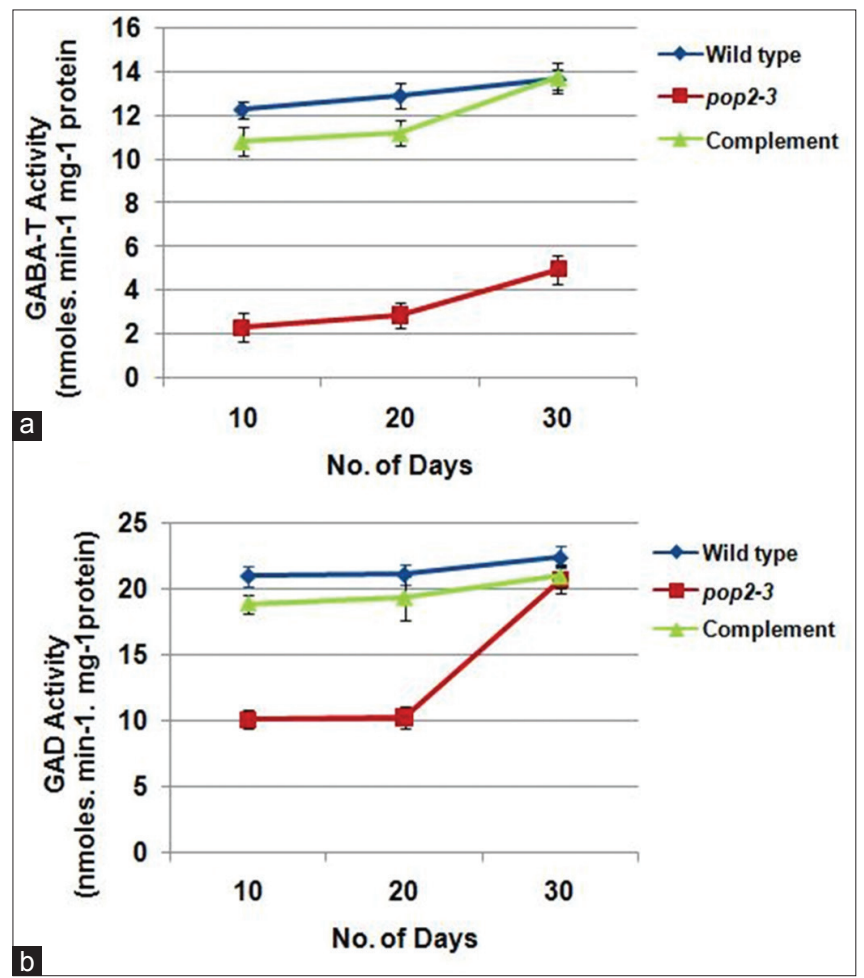

Figure 3: Determination of enzymatic activities of leaves of pop2-3 mutant, gamma-aminobutyric acid transaminase (GABA-T) complement, and wild-type plant of Arabidopsis thaliana, (a) enzymatic activity of GABA-T in pop2-3 mutant, GABA-T complement, and wild type, (b) glutamate decarboxylase activity in pop2-3 mutant, GABA-T complement, and wild-type leaves. Data represent mean \pm standard deviation of 3-5 biological replicates

in intracellular signalling, especially during leaf senescence under stress conditions. T-DNA insertion pop2-3 mutant of $A$. thaliana displays reduced seed production under selffertilization, implying that the role of this enzyme is restricted to flower tissue..$^{[7]}$

GABA-T pop 2-3 mutants act as signals that mediate early leaf senescence. Further, to confirm the role of GABA-T in leaf senescence, we have done the transformation of $A$. thaliana pop2-3 T-DNA insertion mutant with pPZP200GB-GABA-T construct (GABA-T complement plant) that revert to wildtype plant. Similar experiments were performed for the complementation of egyl mutant to ensure the function of ethylene-dependent gravitropism-deficient and yellowgreen 1 in regulation of leaf development and senescence in A. thaliana ${ }^{[27]}$ Several other investigators also performed the same experiment to confirm the role of a respective genes such as TLP 18.3 gene, $^{[12]} A S P 2$ gene, ${ }^{[28]}$ and CYP724A1 gene. ${ }^{[29]}$

We have previously established physiological parameters for the screening of leaf senescence that based on the determination of chlorophyll content, ion leakage, and MDA content at different leaf developmental stages. ${ }^{[15]}$ We have compared the physiological characteristics associated with senescence to the wild-type, pop2-3 mutant, and GABA-T complement plant of A. thaliana. Chlorophyll can provide basic information of photosynthesis, and chlorophyll degradation is an important feature of senescence. Our results showed that chlorophyll contents were low in pop2-3 mutant than wild-type plant after 30 days in normal growth condition. Probably low chlorophyll content in mutant showed precocious leaf senescence [Figure 1a], which was similar to the reported data in egyl mutants, ${ }^{[27]} \operatorname{por} B$ 1por $C-1$ double mutant of older seedlings, ${ }^{[30]}$ and mex 1 leaves of A. thaliana. ${ }^{[31]}$ The chlorophyll content of GABA-T complement plant was approximately similar to wild-type plant up to 30 days [Figure 1a].

During leaf senescence, membrane became fragile, leak, and released many ions in the cell. Ion leakage is an indicator of membrane integrity and important parameter to measure leaf senescence. In this study, ion leakage in pop 2-3 mutant was stable up to 20 days from date of emergence but higher than wild type plant and began to sharply increase from 30 days. However, ion leakage in wild-type leaves stable up to 20 days and slightly increased from 30-day-old plant, whereas there was no increase in the ratio throughout 30 days in GABA-T complement plant and showed approximately similar ion leakage percentage to wild-type plant [Figure 1b]. The result was as similarly found in egyl mutant leaf of A. thaliana. ${ }^{[27]}$

Determination of lipid peroxidation is another parameter to measure the leaf senescence. Our result showed that increases the level of lipid peroxidation and cell damage in pop 2-3 mutant plant in an earlier stage of leaf development as compared to wild type [Figure 1c]. Similarly, the MDA content started to increase at 40 days in wild-type plants but at 30 days in (glutathione reductase 2) igr 2-9 and igr 2-14 mutant's plant of $A$. thaliana. $^{[32]}$ However, GABA-T complement plant showed approximately similar lipid peroxidation level to wild-type plant up to 30 days.

GABA is an important component of GABA shunt, so we determine and compare the GABA content in pop 2-3 mutant with wild-type and GABA-T complement plant. Our result showed that GABA content of pop 2-3 mutant leaves was much higher than wild type plant and was stable up to 20 days sharply increases from 30 days, whereas in wild type, it was approximately stable up to 30 days [Figure 2]. GABA content of GABA-T complement plant was approximately similar to wild-type plant and stable up to 30 days. It has been also reported that ataldh10A8 and ataldh10A9 T-DNA-insertion mutants reduced GABA accumulation than wild-type plants of A. thaliana during salinity treatment and are more sensitive to salinity stress. ${ }^{[33]}$

GABA-T and GAD are the key enzymes in GABA-shunt pathway. Enzymes activities of these enzymes were assayed at different leaf developmental stages in wild-type and pop2-3 mutant plant and compared with GABA-T complement plant. The enzyme activity (GABA-T and GAD) was very 
less in pop 2-3 mutant than in wild-type plants. The enzyme (GABA-T and GAD) activities were stable till 20 days in pop 2-3 mutant, whereas it was stable till 30 days in wildtype plant [Figure $3 \mathrm{a}$ and b]. Analysis of $A t G H B D H$ activity for $A$. thaliana $\gamma$-hydroxybutyrate dehydrogenase, ${ }^{[34]}$ analysis of GABA-T activity in stress conditions. ${ }^{[9,17]}$ The enzyme (GABA-T and GAD) activities of GABA-T complement plant were approximately similar to wild-type plant and stable up to 30 days. Taken together, these results suggested that GABA-T is involved in controlling the onset of leaf senescence in plants.

\section{CONCLUSION}

The mutation of GABA-T gene might release silencing of a subset of critical genes that regulating the leaf senescence process. It appears that GABA-T controls both cellular protection activities and senescence activities. Compare to the wild-type $A$. thaliana, pop2-3 mutant showed low chlorophyll content, increased ion leakage, and increased level of lipid peroxidation than wild-type plant after 30 days in normal growth condition. Probably, these characteristics in pop2-3 mutant showed precocious leaf senescence. A. thaliana pop2-3 T-DNA insertion mutant after transformation with pPZP200GB-GABA-T construct (GABA-T complement plant) revert to wild-type plant and showed similar characteristics of senescence parameters with wild-type plants. Thus, this confirmed that GABA-T is an important regulator of leaf senescence in A. thaliana and plays crucial roles in GABA accumulation and catabolism.

\section{ACKNOWLEDGMENTS}

This work was supported by research grants from Science and Engineering Research Board (SERB), Department of Science and Technology Govt. of India, New Delhi (Grant No. SB/ SO/BB-002/2012), to Dr M. I. Ansari. The authors would also like to acknowledge the Arabidopsis Biological Resource Center (ABRC), Ohio State University of Columbia, USA, for providing the mutant seeds.

\section{REFERENCES}

1. Bouché N, Fromm H. GABA in plants: Just a metabolite? Trends Plant Sci 2004;9:110-5.

2. Pinal CS, TobinAJ. Uniqueness and redundancy in GABA production. Perspect Dev Neurobiol 1998;5:109-18.

3. Owens DF, Kriegstein AR. Is there more to GABA than synaptic inhibition? Nat Rev Neurosci 2002;3:715-27.

4. Represa A, Ben-Ari Y. Trophic actions of GABA on neuronal development. Trends Neurosci 2005;28:278-83.

5. Kinnersley AM, Turano, FJ. Gamma aminobutyric acid (GABA) and plant responses to stress. Crit Rev Plant Sci
2000;19:479-509.

6. Ludewig F, Hüser A, Fromm H, Beauclair L, Bouché N. Mutants of GABA transaminase (POP2) suppress the severe phenotype of succinic semialdehyde dehydrogenase (ssadh) mutants in Arabidopsis. PLoS One 2008;3:e3383.

7. Palanivelu R, Brass L, Edlund AF, Preuss D. Pollen tube growth and guidance is regulated by POP2, an Arabidopsis gene that controls GABA levels. Cell 2003;114:47-59.

8. Ansari MI, Lee RH. A novel senescence-associated gene encoding gamma aminobutyric acid(GABA): Pyruvate transaminase is upregulated during rice leaf senescence. Physiol Plant 2005;123:1-8.

9. Renault H, Roussel V, El Amrani A, Arzel M, Renault D, Bouchereau A, et al. The Arabidopsis pop 2-1 mutant reveals the involvement of GABA transaminase in salt stress tolerance. BMC Plant Biol 2010;10:20.

10. Ansari MI, Chen SC. Leaf senescence-an overview. Int J Recent Trends Sci Technol 2011;1:110-4.

11. Shelp BJ, Bown AW, McLean MD. Metabolism and functions of gamma-aminobutyric acid. Trends Plant Sci 1999;4:446-52.

12. Ansari MI, Lin TP, Liu MS. Molecular characterization of TLP 18.3 gene of Arabidopsis thaliana. Int J Integr Biol 2011;11:44-51.

13. Narayan VS, Nair PM. Metabolism, enzymology and possible roles of $\mathrm{g}$ amino butyrate in higher plants. Phytochemistry 1990;29:369-75.

14. Ansari MI, Chen SC. Biochemical characterization of gamma-aminobutyric acid (GABA): Pyruvate transaminase during rice leaf senescence. Int $\mathrm{J}$ Integr Biol 2009;6:27-32.

15. Jalil SU, Ahmad I, Ansari MI. Functional loss of GABA transaminase (GABA-T) expressed early leaf senescence under various stress conditions in Arabidopsis thaliana. Curr Plant Biol 2017. DOI: 10.1016/j.cpb.

16. Miyashita Y, Good AG. Contribution of the GABA shunt to hypoxia-induced alanine accumulation in roots of Arabidopsis thaliana. Plant Cell Physiol 2008;49:92-102.

17. Al-Quraan NA, Al-Share AT. Characterization of the $\gamma$-aminobutyric acid shunt pathway and oxidative damage in Arabidopsis thaliana pop 2 mutants under various abiotic stresses. Biol Plant 2016;60:132-8.

18. Chu CC, Lee WC, Guo WY, Pan SM, Chen LJ, Li HM, et al. A copper chaperone for superoxide dismutase that confers three types of copper/zinc superoxide dismutase activity in Arabidopsis. Plant Physiol 2005;139:425-36.

19. Clough SJ, Bent AF. Floral dip: A simplified method for Agrobacterium-mediated transformation of Arabidopsis thaliana. Plant J 1998;16:735-43.

20. McDowell JM, Dhandaydham M, Long TA, Aarts MG, Goff S, Holub EB, et al. Intragenic recombination and diversifying selection contribute to the evolution of downy mildew resistance at the RPP8 locus of Arabidopsis. Plant Cell 1998;10:1861-74. 
21. Arnon DL. Complex enzymes in isolated chloroplasts: Polyphenol oxidase in Beta vulgaris. Plant Physiol 1949;24:1-15.

22. Chen GH, Liu CP, Chen SC, Wang LC. Role of Arabidopsis a fifteen in regulating leaf senescence involves response to reactive oxygen species and is dependent on ethylene insensitive2. J Exp Bot 2011;62:1-18.

23. Heath RL, Packer L. Photoperoxidation in isolated chloroplasts. I. Kinetics and stoichiometry of fatty acid peroxidation. Arch Biochem Biophys 1968;125:189-98.

24. Miflin BJ, Habash DZ. The role of glutamine synthetase and glutamate dehydrogenase in nitrogen assimilation and possibilities for improvement in the nitrogen utilization of crops. J Exp Bot 2002;53:979-87.

25. Ansari MI, Hasan S, Jalil SU. Leaf Senescence and GABA Shunt. Bioinformation 2014;10:734-6.

26. Jalil SU, Ahmad I, Ansari MI. Screening of Arabidopsis thaliana GABA-transaminase mutant and its root pattern analysis. J Biol Chem Res 2016;33:909-17.

27. Chen C, Wang J, Zhao XX. Leaf senescence induced by EGY1 defection was partially restored by glucose in Arabidopsis thaliana. Bot Stud 2016;57:5.

28. Brauc S, de Vooght E, Claeys M, Höfte M, Angenon G. Influence of over-expression of cytosolic aspartate aminotransferase on amino acid metabolism and defence responses against Botrytis cinerea infection in Arabidopsis thaliana. J Plant Physiol 2011;168:1813-9.

29. Zhang R, Xia X, Lindsey K, da Rocha PS. Functional complementation of dwf4 mutants of Arabidopsis by over expression of CYP724A1. J Plant Physiol 2012;169:421-8.

30. Frick G, Su Q, Apel K, Armstrong GA. An Arabidopsis porB porC double mutant lacking light-dependent NADPH: Protochlorophyllide oxidoreductases B and Cis highly chlorophyll-deficient and developmentally arrested. Plant J 2003;35:141-53.

31. Stettler M, Eicke S, Mettler T, Messerli G, Hörtensteiner S, Zeeman SC. Blocking the metabolism of starch breakdown products in Arabidopsis leaves triggers chloroplast degradation. Mol Plant 2009;2:1233-46.

32. Ding S, Wang L, Yang Z, Lu Q, Wen X. Decreased glutathione reductase2 leads to early leaf senescence in Arabidopsis. J Integr Plant Biol 2016;58:29-47.

33. Zarei A, Trobacher CP, Shelp BJ. Arabidopsis aldehyde dehydrogenase 10 family members confer salt tolerance through putrescine-derived 4-aminobutyrate (GABA) production. Sci Rep 2016;6:35115.

34. Breitkreuz KE, Allan WL, Van Cauwenberghe OR, Jakobs C, Talibi D, Andre B, et al. A novel gammahydroxybutyrate dehydrogenase: Identification and expression of an Arabidopsis cDNA and potential role under oxygen deficiency. J Biol Chem 2003;278:41552-6.

Source of Support: Nil. Conflict of Interest: None declared. 